Research Article

\title{
Acceptance of Information and Communication Technologies for Healthy and Active Aging: Results from Three Field Studies
}

\author{
Filomena Papa *, Bartolomeo Sapio, Enrico Nicolò
}

Fondazione Ugo Bordoni, Viale del Policlinico 147, 00161 Roma, Italy; E-Mails: fpapa@fub.it; bsapio@fub.it; nic@fub.it

* Correspondence: Filomena Papa; E-Mail: fpapa@fub.it

Academic Editor: Lisa Hollis-Sawyer

Special Issue: Got Aging? Examining Later-life Development from a Positive Aging Perspective

\section{OBM Geriatrics}

2019 , volume 3 , issue 1

doi:10.21926/obm.geriatr.1901028
Received: October 31, 2018

Accepted: December 19, 2018

Published: January 04, 2019

\begin{abstract}
Background: Information and Communication Technologies (ICTS) have the potential to promote healthy aging and increase the quality of life of older adults. However, several barriers like access, performance, psychological, and privacy issues still exist against fully deploying ICT solutions for older adults. To determine useful methods to overcome such barriers, this work investigated the possible factors that prevent elders from directly accepting ICT services based on three field studies.
\end{abstract}

Methods: The Unified Theory of Acceptance of Use of Technology (UTAUT) was adopted as the general reference framework.

Results: Performance Expectancy (perceived usefulness) and Facilitating Conditions (provided user support) seem to be the most relevant factors in all field studies. Also, the relevance of Hedonic Motivation (pleasantness of use) emerges. The role of Effort Expectancy (ease of use) seems to be affected by the user profile, which includes education level, present technology skill, and employment status.

Conclusions: Overall, results confirm that the reference model variables represent the key factors to predict older adults' viewpoints toward ICT. The use of familiar devices (like TV sets) in the interaction with new technologies was determined to be a significant factor in

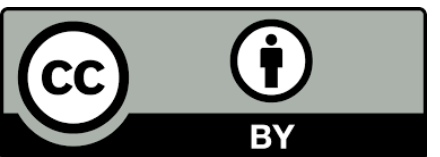

(C) 2019 by the author. This is an open access article distributed under the conditions of the Creative Commons by Attribution License, which permits unrestricted use, distribution, and reproduction in any medium or format, provided the original work is correctly cited. 
promoting ICT acceptance by older adults. Furthermore, acceptance/rejection of ICT services depends on classical socio-demographic variables and on participants' attitude towards technology, lifestyle changes, and the beliefs that individuals share with members of their social context.

\section{Keywords}

Effort expectancy; electronic health record; facilitating conditions; healthy aging; hedonic motivation; performance expectancy; technology acceptance; unified theory of acceptance and use of technology (UTAUT)

\section{Introduction}

Information and Communication Technologies (ICTs) can provide useful and effective tools to meet the expectations of people to live longer higher quality lives. Current technological trends in health care are focused on technological solutions to support disease management and medical records. ICTs may also enhance communication between participants using the technology, which plays a significant role in their social capabilities and mental well-being,

Designing ICTs for older adults requires specific understanding of their needs and comparative analysis of the generational use of new media and technology [1].

In a recent study by the Italian Statistics Institute (ISTAT) [2], statistics on Internet use show that $30.8 \%$ of people $65-74$ years old and $8.8 \%$ of people over 75 have used the Internet during the last year.

Several barriers like access, performance, psychological, and privacy issues still hinder the full deployment of ICT solutions for older adults [3]. In particular, rejection attitudes toward new ICT services and social media can arise, especially in groups with low income and poor education [4]. In order to overcome these barriers, the investigation of factors that affect older adults from accepting ICT services provides useful insight to identify adequate policies to promote acceptance. One of the most recognized models for innovative and emerging new technologies is the Unified Theory of Acceptance and Use of Technology (UTAUT), which is extensively used in various applications, such as technology use in different organisational contexts [5], consumers' use of technology, e-commerce. This model is comprised of different constructs, including intention to use ICT services, perceived usefulness, and perceived ease of using ICT services. Implementing the UTAUT model as a general reference framework, this paper discusses the factors affecting acceptance of ICT services by older adults. Results were obtained from three field studies, which focused on the acceptance by older adults through direct involvement with different ICT technologies (i.e., a TV-based technological solution for social interaction, an Electronic Health Record, Digital Television) and implemented the same reference framework.

\subsection{Field Study 1}

This study analyzed the use of ICTs to support social interactions of older adults which are defined in this case as $65+$ years of age. The virtual world via the Internet has potential to mitigate 
social isolation problems faced by seniors. This work presents a qualitative investigation of the reactions, opinions, and sentiments about a TV-based technological solution to promote social interactions of less educated older adults (i.e., older adults unskilled in ICT use with low education and low income). Experimental data were collected by extensive trials involving 40 end users in the European AAL project EasyReach. A methodology called "scenario engagement" was applied to engage participants in a live demonstration, which was mediated by a facilitator who assisted older adults to interact with the system.

\subsection{Field Study 2}

This study relates to the use of ICT solutions to maintain the physical well-being of older adults, to promote the use of self-monitoring technologies for patients with chronic illnesses, and to better prevent the loss of independence and good health. The acceptance by citizens of one of the most advanced implementations of the Electronic Health Record (EHR) in Italy is investigated in this study. The system provides applications for self-measurement, inputting drug treatments, entering examinations conducted at laboratories, downloading and viewing medical reports as soon as they are issued, online payments, virtual prescriptions. The UTAUT model and its extension UTAUT2 were adopted as general reference frameworks. The quantitative investigation was conducted using the CAWI (Computer Assisted Web Interview) method, with a total number of 15,102 users completing the web interview. Among these citizens, 5,664 were over 55 years old.

\subsection{Field Study 3}

This field study explores the interaction of older adults with new television services and new ICT services. TV services are a central source for elders and could contribute to maintaining their mental well-being. Furthermore, the use of familiar devices (like TV sets) combined with ICT services could promote the adoption of these services by older adults. This qualitative study performed in Italy is focused on the acceptance of Digital Terrestrial Television (DTT) after the analogue switch-off, that is the transition from analogue to digital terrestrial television. In order to identify the main predictors of digital television usage by older adults, the UTAUT model was applied, followed by a focus group discussion analysis.

In Section 2, the adopted reference frameworks are described (UTAUT and UTAUT2). In Sections 3-5 the three field studies are presented, and Section 6 discusses the factors affecting acceptance of ICT services by older adults.

\section{The Reference Framework}

From the point of view of the ICT users, the decision to accept new technologies is an innovative step and is based on two conditions: if the technology is useful to them, and if they have the skills, means, and situation to use the presented technology. Some reasons for innovative change include the natural curiosity to try new things and social influence by significant others who push them to innovation, such as family, friends, or bosses [6]. Understanding the social dynamics of ICT might help engineers to focus technological designs on meeting the users' demands. Previous studies have reported the role of domestication of Information and Communication Technologies in depth and its influence on usage by elders $[7,8]$. 
User experience is a very broad concept dealing with all aspects of a user's interaction with the product/service, including how it is perceived, learned, and used [9]. Behind the user experience concept, there is a holistic approach aimed at balancing the pragmatic aspects mainly related to the execution of the task- and non-task-related aspects. Examples of these include aesthetic, psychological, and emotional aspects like self-expression through technology use [10].

The evaluation of different user experience aspects can be utilized as input for user acceptance models. These models are powerful tools to explain individual acceptance and usage of a technological system. They also allow the identification of most relevant factors that facilitate and hamper usage (see, for instance, [11]).

The Unified Theory of Acceptance and Use of Technology (UTAUT) [5] is one of the most recognized acceptance models. This model, comprising different aspects of usage behavior, has been extensively implemented to explain the adoption and use of various innovative ICT products and services.

The UTAUT can explain usage behavior of an innovative technology or service starting from the central role of behavioral intention. The key dependent variable in the UTAUT model is the intention or the use of technology; hence, the primary purpose of the model is to understand and provide an explanation for the phenomenon "use of information technology" as a dependent variable. The role of intention, as an antecedent of behavior, is a critical factor in the model. In the formulation of the UTAUT model, four constructs are identified, which may play a significant role as direct determinants of user acceptance and usage behavior:

- Performance Expectancy is the degree to which an individual believes that using the system will help him/her to attain gains in job performance.

- Effort Expectancy is the degree of ease associated with the use of the system, which is strongly connected to the degree to which a person believes that using the system would be free of effort.

- Facilitating Conditions are related to the degree to which an individual believes that an organization or technical infrastructure exists to support the use of the system.

- Social Influence is the degree to which an individual perceives that significant others, such as spouses and family members, believe they should use the new system.

In the specific case of technology designed for an aging population, it is possible to measure the above-mentioned constructs using some of the following indicators:

Performance Expectancy (perceived usefulness of services):

- Degree of interest of the user towards provided services and content;

- Update of provided content;

- Service interaction and fruition modalities;

- Service capability to attract and entertain the user, avoiding banality and tediousness in the interaction;

- Satisfaction of using service in comparison to the same kind of service delivered through other channels.

Effort Expectancy (perceived ease of use): 
- Ease of access to the services;

- Ease of system use;

- Ease of navigation;

- Perception by the users of possible system dysfunctions (excessive waiting times, unclear costs, etc.).

Social Influence:

- Attitude towards technology of the persons who are considered important by the user (e.g., affective and charismatic leaders);

- Behavior towards technology of own reference groups (e.g., friends, associations).

Facilitating Conditions (provided user support):

- User manuals;

- Call center or contact center.

Variables, such as age, gender, experience, and voluntariness of use, are important moderators included in the UTAUT model. Income and education have been proposed as moderators to be validated in future studies [11].

More recently, the UTAUT2 model was proposed as an extension of the UTAUT model. UTAUT2 can be utilized in larger contexts and includes new constructs connected to habit, price value, and hedonic motivation in use [12].

Furthermore, in the particular case of e-health, it is necessary to consider an additional construct [13] concerning security perception, privacy protection, and personal data confidentiality.

\section{Field Study 1: TV Set to Overcome Barriers for Older Adults in ICT Use}

Assuming that the ability to use a TV remote control can be exploited as a sufficient factor to become effective users of new digital social environments and to reduce rejection attitudes, a qualitative investigation on the reactions of older adults toward a TV-based technological solution to promote social interaction was carried out. The investigation was developed by the EasyReach Project (AAL-2009-2-117) under the EU Seventh Framework Program (Ambient Assisted Living Joint Program, AAL Call 2). A technological solution was implemented, which aimed at fostering social interaction for individuals within the aging population who are resistant to the adoption of new technology or lack the knowledge and skills needed to use social networks. A special interactive environment was created to enhance the social contact of aged people and to respond to their needs by using a "traditional" technology that does not introduce any further resistance to the acceptance of the innovation.

The user-centered design approach [14] was adopted to directly involve less educated older adults (unskilled in ICT use, low education, and low income) in the early stages of the development process. A pilot investigation was carried out in Rome, Italy, involving real users [15].

The use of ICTs in health care could allow disadvantaged groups of citizens, such as elderly, chronically ill people, or those living with various physical and cognitive disabilities, to maintain 
vital and rich connections with families, friends, and careers. ICTs can help them keep in touch and socialize through social network applications [3].

The field of studies related to Social Network Sites (SNS) concerns the use of ICTs to support social interactions of people through SNSs. The virtual world and social networks are potentially important to meet elderly persons' needs, who can subsequently create and develop relationships with other older adults and members of different backgrounds and generations. SNSs have the potential to mitigate social isolation problems felt by seniors and to help them maintain their social networks [16].

Despite the many potential benefits for elderly socialization, a base core of knowledge and skills is needed to use social networks, which is not widespread in the community of less-educated older adults. Few studies have been developed in the area of socialization applications for unskilled older adults. For example, an investigation [17] developed in the framework of the European AAL research program utilized a Smart TV platform to support social interaction of older adults with their relatives and other people sharing the same interests. The approach was to use well-known (by older adults) existing technology to avoid rejection attitudes and to provide a solution to mitigate social isolation of older adults. Following a similar approach, another study [18] revealed that when technology is embedded in everyday objects, such as a TV set, older people can use it easily.

\subsection{The EasyReach Solution}

The main hypothesis in designing the EasyReach solution was that for less-educated aged people, who do not possess the necessary skills to use the Internet and social networks, daily experience with TV and remote control could be a key qualification to become an effective user of those services. Moreover, a TV-like system may help overcome the psychological barriers and the rejection attitudes toward new ICT services and social media. In comparison with the abovementioned studies, using similar approaches, the EasyReach technological solution utilizes a new kind of remote control that combines gesture recognition with video and audio capture capabilities.

The key idea of EasyReach is to maintain simplicity for users, in both system installation and utilization. It is based on a special social TV channel accessed by older adults through their own TV set, a set-top box and a specialized remote control unit endowed with gesture recognition and video and audio capture capabilities [19]. A hidden personal assistant (PA) elaborates user preferences in the background, allowing better focalization on his or her social interests.

After connecting the set-top box to the TV set, the user is immediately able to join the social channel and enjoy the benefits of networking.

With the EasyReach special remote control, the user can take pictures and videos of special events (e.g., a family dinner, a birthday party, etc.), share his or her knowledge and experience by creating a video message and posting it on thematic groups, and simply have conversations with friends and relatives. The remote control's gesture recognition mechanism helps the user navigate by giving him/her the possibility to scroll a personal list of users and groups through simple gestures.

Moreover, the system allows organizations and official institutions to join the social channel in order to be easily reachable by the users, send them useful information/advice, and help them 
more directly. The opportunity to make the user's own expertise available to the whole EasyReach community can be invaluable to maintain the self-perception of being socially active.

\subsection{Method}

The main requirements about elderly participants as users can be shortly summarized into factors: "poor education," "ICT unfamiliar," "low income," and "resistant to change." These four factors strongly influence the technical solutions and the way to select the potential participants. In this work the research team analyzed different possible alternatives, especially in view of the direct involvement of users in field trials and their reactions toward the proposed technical solution. Two senior centers in Rome were selected [15]. Preliminary sessions to better understand end users' reactions and behaviors were purposely organized. These early trials quickly revealed many unpredictable aspects. It was recognized that applying any quantitative metrics to collect data was an impossible task, mostly due to participants' resistance and ungovernability during the sessions. The early sessions also helped design a pilot system capable of collecting effective users' reactions, opinions, and sentiments about the EasyReach system, including ease of use and usefulness. User experience and acceptance models were taken as broad references.

Laboratory testing revealed a lack of fluidity in interaction with the system, resulting in a frustrating experience for users. Because gestures to browse the interface were not very easy to learn, the project team decided to set up a sort of "mediated experience" for the older adults during the pilot using an original methodology called "scenario engagement" [15]. This approach takes into account the strong support needed by older adults and their interaction with different technologies [20]. The choice was to analyze user testing [21], in which participants engaged in a live demonstration of the EasyReach solution with the support of a facilitator.

A "scenario engagement" methodology for the execution of the field evaluation is defined as the intent of carrying out an assessment and the "acceptance towards the idea of..." or the "what if..." This methodology was implemented after the involved older adults were engaged in a live demonstration illustrating scenarios of possible use.

Implementing scenario engagement, a live demonstration performed under certain procedural settings can be equally effective to assess the users' reactions and their own experiences in interacting with the system. Even though the users can control the system, they are guided stepby-step by a mediator supporting them in the manipulation of devices.

\subsubsection{Participants}

A screening questionnaire was administered to select participants for the qualitative investigation.

Two senior centers, representing two different geographical and cultural areas in Rome, were selected for the pilot test in cooperation with Federazione Nazionale Pensionati (FNP), the retired and elderly federation of CISL (trade union).

Presentation meetings with older adults were organized in each of the two senior centers in September 2012. During these meetings, the screening questionnaire was administered to participants. 
The criteria used in the selection process were related to age (preferably in the range 65-80), education (preferably "primary" and "secondary" education), and computer and Internet usage (preferably no more than once per month). Physical characteristics of participants, such as visual and hearing capabilities, were not considered in the selection.

Forty participants ( 21 males, 19 females) were recruited for the pilot test (30 individuals from the two senior centers, 15 for each center, plus 10 others selected from FNP-CISL as "privileged witnesses" of the elderly world).

Demographic data of participants showed a balanced distribution of genders $(52 \%$ males and $48 \%$ females) with a peak age range of 66-70 (32\% of total participants). Most of them had a low level of education ( $80 \%$ in the "primary - secondary" education range). Almost all participants did not use computers (85\%) or the Internet $(72 \%)$.

\subsubsection{Procedure}

The field trial was realized by setting up group sessions at the senior center premises involving the selected users. About 10 users were involved in each session. A total of four group sessions were realized with two sessions at each senior center in 2013.

Each session was developed through three main segments: a demonstration of the main features of the EasyReach system (remote control, user interface); scenario engagement of the older adults in tasks with the EasyReach prototype, such as planning a group activity (a "mediated user experience" was setup); and collection of the users' opinions. The duration of the overall group session was about 80 min.

The usage scenarios were carefully rehearsed to avoid time consuming or likely-to-fail actions. User profiles and user groups identified in the scenarios were created in the set-top boxes, and good quality videos and photos were uploaded offline into the "galleries" of selected users.

During the trial sessions, two simple activities were identified, which fit the usual life habits of participants from the two senior centers. Two different scenarios were demonstrated, showing the added value of EasyReach utilization in comparison to traditional communication media (e.g., phone calls).

The theme of the first scenario engagement was the organization of a group activity: "A Day to The Cinema." The theme of the second scenario was the communication of good news to friends: "Becoming a Grandmother."

One researcher conducted the session using a printed script, while a second researcher (facilitator) interacted with the system during the group session. Another researcher (recorder) took note of the most relevant issues emerging from the interview.

User opinions were collected, which concerned the following issues: perception of usefulness, pleasantness of use, and ease of use of the EasyReach solution; intention of future service usage in different environments (at home and at the senior center) when system is available; and the potential for social inclusion and improvement in quality of life.

General impressions about the system, its main features/service, and suggestions for improvement were also investigated.

In the last part of the session, a group interview was realized to collect user opinions about the EasyReach system. 
In addition to the group interview, personal interviews were administered to 10 "privileged witnesses" to analyze the potential of the EasyReach solution.

Both group and personal interviews were audio and video recorded. Transcriptions of group and personal interviews were made, then content analysis was conducted to point out the most relevant emerging topics.

\subsubsection{Ethical Considerations}

Although a formal ethical approval was not explicitly required by the AAL program, a targeted analysis of the ethical issues was included in the EasyReach work plan. After the preparation phase of the pilots, the following aspects were highlighted: privacy protection and confidentiality, informed consent/disclaimers, and risk assessment. Formal discussions taken inside the consortium produced a general agreement about the policy and ethical guidelines to be observed before, during, and after the pilots. Both the EasyReach meeting notes (Project Steering Committee, Technical Management Board) and Deliverable D6.1 "Detailed Plan for the Pilots" [22] provide proof of the work developed on the matter.

In particular, the executive team of the Rome pilot included two extra researchers who supervised the application of ethical guidelines.

The EasyReach work plan approved by the AAL program, as required, included an Ethical Issues Table. In this table, it was stated that the research involves "adult healthy volunteers" and that "all country specific provisions, specific laws and regulations will be respected." Also in the Rome pilot, the regulations for personal data protection (law no. 186, 30 June 2003) were respected. A formal ethical approval by a recognized authority was not explicitly required by the AAL program for funding this research.

As stated in the EasyReach work plan, informed consents were submitted to end users in advance. They were informed of the aim to study that specific population, the voluntariness in the participation, the possibility to withdraw at any moment, the policy for anonymity, the expected benefits/risks of this research, how long the confidential information would be kept, and the indication of a reference person to contact for any questions related to the research.

Each participant in the Rome pilot was requested to compile a disclaimer (i.e., a "release form for media recording"), in which the participant was informed that "the experimental activities and discussions will be recorded and some photos will be taken." In this form, the participants were asked to express the "agreement for the diffusion of the audio and video materials obtained during the sessions through different channels such as scientific papers, conferences, TV programs, internet, etc." All participants in the Rome pilot signed the above disclaimer as requested and returned the signed form to the research team.

\subsubsection{Data Analysis}

The user acceptance UTAUT model was adopted as the theoretical framework for investigation and data analysis [5] in association with the concepts of quality of life and perceived loneliness.

In the investigation, the perception of improved quality of life was included in the section of the UTAUT model related to the perception of usefulness of the system. We focused the group interviews on themes related to perceived usefulness (Performance Expectancy), perceived pleasantness and ease of use (Effort Expectancy), and intention of use (Behavioral Intention). The 
Facilitating Conditions in this investigation were part of the scenario engagement methodology, thus we decided not to investigate this issue. We decided not to investigate social influence since this theme was a sensitive topic to discuss with this particular class of older adults.

From the group interviews, about $100 \mathrm{~min}$ of video/audio registrations were obtained.

From the personal interviews to "privileged witnesses," about $300 \mathrm{~min}$ of video/audio registrations were obtained. Qualitative content analysis was executed using both notes and transcriptions.

This analysis was developed by researcher members of the team through three steps: (1) reading notes and transcriptions, (2) identifying relevant opinions/sentences, and (3) making comments. The results were consolidated in group discussions involving all members of the research team. More details about data analysis procedures can be found in [15].

\subsection{Results}

The investigated solution can be effectively employed to foster social interaction, particularly when it is introduced in a collective use environment (e.g., senior center). Although the focus of the investigation was on information and communication technology-enabled social environments, the end users themselves strongly suggested that future systems include extra functions, as essential opportunities for their potential digital lives, meaning medical or health services and bridges toward Public Administrations.

The pleasantness and ease of use were also carefully examined as potential factors that may affect usage. In general, the system was considered to be simple and pleasant to use, except for the dislike of the specific remote control.

The intention to use the system as a tool to enhance social communication was also investigated. A wide range of usage intentions was gathered which span from negative to neutral to enthusiastic attitudes. Future suitable scenarios of use for this solution are likely to favor the collective environment (at the senior center) rather than a solitary use from home. Technologyresistant older adults asked for strong support and help, from which an environment allowing a collective use seems to be advisable.

Despite the difficulties in direct interaction (i.e., slowness and inadequacy of the remote control), personal interviews to "privileged witnesses" showed great potential for social inclusion and the great capacity of EasyReach to improve the quality of life of aging people. The system seemed to stimulate the participants' curiosity as well to open their mental horizons to further possibilities that can facilitate their daily lives.

\section{Field Study 2: Older Adults' Acceptance of e-Health Services}

\subsection{Context}

This study introduces a quantitative investigation about the acceptance by citizens of one of the most advanced implementations of the Electronic Health Record (EHR) in Italy. From a national perspective, the EHR initiative aims at improving all care activities throughout the patient's life. Therefore, the dossier is continually fed by professionals within the Italian National Health Service (SSN) and regional services, as well as at the request of the citizens with the medical records held by them. 
The improvement of health services and care activities is perceived very important by older adults. Medical or health related services have high recurrence among elderly needs and requirements to increase their quality of life [15]. To satisfy the needs of older adults the EHR could provide potential benefits [23].

The regional EHR presented here, TreC service (literally, Citizens' Clinic Record), is promoted by the Trento Autonomous Province in Italy.

This exploratory study allows researchers to investigate factors that facilitate or hamper the acceptance of TreC services by citizens and to compare the results obtained for the overall sample of citizens containing the group of adults over 55. At the same time, it can provide useful input to define specific public policies to promote acceptance of innovative e-health solutions by older adults.

\subsection{Method}

\subsubsection{The TreC System}

The system was designed to be a configurable platform of applications and initially included a series of functions for archiving and codifying healthcare data and applications for selfmeasurement, inputting drug treatments, entering examinations conducted at laboratories outside the Region, and so on. In addition, from the outset, the infrastructure offered the opportunity to download and view medical reports as soon as they were issued by laboratories. Later, other services, such as online payments, virtual prescriptions and other minor services were made available [24].

\subsubsection{Data Collection and Participants}

From the time of its birth, the TreC service was monitored by means of quantitative and qualitative studies planned and developed by the Department of Sociology and Social Research of the University of Trento for the entire duration of the project (2008-2013).

This investigation is based on one activity in particular, a CAWI (Computer Assisted Web Interview) addressed to approximately 34,000 active users registered with the infrastructure at the end of the fourth year of operation (2016).

A specific section of the CAWI was finalized to measure acceptance constructs (see Section "Investigated issues").

A total number of 15,102 users completed the web interview, 5,664 of which were over 55 years old. Participants belonged to different classes of gender, age, education, and employment. Overall, the average participant profile was of middle age, high education (degree or post degree), skilled in technology use, and employed [25].

\subsubsection{Investigated Issues}

To investigate the factors affecting acceptance by citizens of the TreC system, the UTAUT2 model (see Section 2) was used as a broad reference. In the case of e-health and electronic health records, we considered all four constructs of the original UTAUT model (Performance Expectancy, Effort Expectancy, Social Influence, and Facilitating Conditions) and Hedonic Motivation from 
UTAUT2. Price Value was not considered because TreC users did not pay for the service, and Habit was also excluded due to the limited experience users may have with the system. The additional construct concerning Security Perception, connected to perception of privacy protection and personal data confidentiality provided by the TreC system, was included.

As described in Section 2, the construct "Behavioral Intention" was adopted to measure acceptance.

The items included in the questionnaire to measure each construct are summarized in Table 1.

Table 1 Items of the questionnaire

\begin{tabular}{ll}
\hline Construct & Item \\
\hline Performance Expectancy (PE) & PE1 The TreC system is useful in my every day life \\
& PE2 The TreC system helps me to manage my health \\
& PE3 The TreC system allows me to save time \\
Effort Expectancy (EE) & EE1 Learning how to use TreC is easy for me \\
& EE2 After the first accesses, TreC is easy to use \\
Perceived Security (PS) & PS1 TreC protects confidentiality of my personal data \\
Social Influence (SI) & SI1 My relatives/friends think that it is useful for me to use \\
Hedonic Motivation (HM) & TreC \\
Facilitating Conditions (FC) & FC1 I have the technological resources to use TreC \\
& FC2 I have the knowledge necessary to use TreC \\
& FC3 I can get the necessary help when I have difficulties using \\
& TreC \\
Behavioral Intention (BI) & BI1 I intend to continue using TreC in the future \\
& BI2 I would like to continue using TreC more and more
\end{tabular}

All items were measured using a four-point Likert scale with the anchors being "strongly disagree" and "strongly agree."

\subsubsection{Ethical Considerations}

The ethical approval must be requested for all researches foreseeing experiments involving human beings, which imply a risk for the psychological and physical well-being of the participants. As was not the case for this research program, researchers applied the art. 110 of Italian Privacy Law (DL 186/2003 - Privacy in medical, Biomedical and epidemiology research). The invitation reported that the participation in the study was voluntary. Participants were clearly informed of the aim to study the specific user's population, voluntariness in the participation, possibility to withdraw at any moment, policy for anonymity, expected benefits/risks of this research, and indication of two reference persons to contact for any questions related to the research. An email address was provided for any request of information or for any communication about the inclusion/non-inclusion in the list used for the research. 


\subsection{Results}

As the first step in data analysis [25], the reliability (internal consistency) of multi-item scales was evaluated. The Cronbach's Alpha values were all greater than 0.7 , suggesting that all adopted scales can be considered reliable.

In order to investigate the factors affecting acceptance, multiple linear regression analysis was adopted, using the assessment of Behavioral Intention (BI) as the dependent variable and the assessment of PE (Performance Expectancy), FC (Facilitating Conditions), HM (Hedonic Motivation), PS (Perceived Security), SI (Social Influence), and EE (Effort Expectancy) as predictors.

In Table 2 the results of the regression analysis are summarized.

Table 2 Results of the multiple linear regression analysis (depending variable: Behavioral Intention)

\begin{tabular}{l|lll} 
Predictors & Beta (standardized) & $t$ & $p$ \\
\hline PE (Performance Expectancy) & 0.260 & 33.152 & 0.000 \\
FC (Facilitating Conditions) & 0.226 & 28.438 & 0.000 \\
HM (Hedonic Motivation) & 0.184 & 21.711 & 0.000 \\
PS (Perceived Security) & 0.102 & 14.675 & 0.000 \\
SI (Social Influence) & 0.076 & 10.247 & 0.000 \\
EE (Effort Expectancy) & 0.016 & 2.014 & 0.044
\end{tabular}

$\mathrm{N}=15102$, Overall model: $\mathrm{R}$-Square $=0.415, \mathrm{p}<0.044$

The regression model explains $41.5 \%$ of the variance in the intention to use TreC.

As shown in Table 2, all coefficients were significant ( $p<0.000$ for PE, FC, HM, PS and $\mathrm{SI} ; \mathrm{p}<0.044$ for EE). The positive dependencies emerging from the analysis indicate that the more subjects perceived high values of these predictors, the more positive the evaluation of the intention was to use TreC in the future. Performance Expectancy and Facilitating Conditions (Beta=0.260 for PE and Beta $=0.226$ for $\mathrm{FC}$ ) appear to be the most relevant determinants of the Behavioral Intention to use the TreC system.

Other predictors of the intention to use TreC are Hedonic Motivation (Beta=0.181) and Social Influence (Beta=0.076). Hedonic Motivation is mainly related to the pleasantness of using TreC. Social Influence is connected to the social context of the user (opinions of relatives and friends).

In predicting $\mathrm{Bl}$, Effort Expectancy (Beta $=0.016$ for $\mathrm{EE}$ ) appeared to be of minor importance.

In Table 3 the results of the multiple regression analysis for the sample of adults over 55 are shown.

In summary, all predictors are significant with the exceptions of Social Influence and Effort Expectancy. The model does not have any integration by these predictors, which is probably a consequence of the recruitment. 
Table 3 Multiple linear regression analysis (depending variable: Behavioral Intention): adults $55+$

\begin{tabular}{l|lll} 
Predictors & Beta (standardized) & $t$ & $p$ \\
\hline PE (Performance Expectancy) & 0.273 & 16.863 & 0.000 \\
FC (Facilitating Conditions) & 0.236 & 14.283 & 0.000 \\
HM (Hedonic Motivation) & 0.197 & 11.457 & 0.000 \\
PS (Perceived Security) & 0.116 & 7.618 & 0.000 \\
SI (Social Influence) & 0.028 & 1.700 & 0.089 \\
EE (Effort Expectancy) & 0.020 & 1.147 & 0.25 \\
$\mathrm{~N}=5664$, Overall model: R-Square $=0.392, \mathrm{p}<0.000$ & &
\end{tabular}

\section{Field Study 3: Older adults' Acceptance of Digital Television}

User experience during transition from analogue to digital terrestrial television was presented in [26], focusing on older adults (age range from 70 to 79).

We performed a qualitative investigation to explore and analyze user experience with digital television and, more in general, with future services made available by broadband ICTs.

The results showed that the impact of the change from analogue to digital television was very strong. The investigation suggested that older adults who extensively watch new digital television channels were the same using their explorative abilities over the Internet (reading online newspapers or looking for old friends on Facebook).

More details about the portion of the study regarding older adults are provided in the following sections.

\subsection{Context}

The analysis of the transition process to digital terrestrial television in several European countries shows that older adults are unequivocally part of the population that meets the greatest difficulties and problems in the management of Digital Terrestrial Television (DTT) [27].

Numerous studies have been conducted on the use of ICT by older people [28]. However, there are few systematic surveys of the user experience with DTT and the user experience of older people with digital TV services [11, 29].

The investigation was based on the direct involvement of older adults and was mainly carried out by using the focus group technique.

The investigation was developed in the framework of the project "Monitor DTT" (Monitoring user experience with DTT) carried out by Fondazione Ugo Bordoni for the Italian Ministry of Economic Development (Department of Communication).

In this survey, the following main aspects of the user experience were considered: 
- Need and use of DTT compared to other media (e.g., Internet, satellite, or mobile television);

- Context of use (situations of use, social dynamics, social and individual TV viewing, and multitasking);

- Interaction with DTT equipment (remote controls, technological integration, impact of the equipment at home, etc.);

- Perception of the advantages and usefulness of the DTT (for example, better image quality, more programs and content, pay TV);

- Expectations on DTT and future ICT services.

\subsection{Methodology}

A qualitative approach was adopted to explore the phenomenon and construct hypotheses to understand the user's "point of view," behaviors, emotions, representations, needs, and future needs [30].

For this survey, in-depth interviews and focus groups were carried out.

The project started in July 2010 by interviewing two experts in the field external to the research team, in order to analize the issues to be explored during the focus groups.

After the interviews, a focus group was held in September 2010 with senior users (70-79 years). This focus group lasted two hours and consisted of 10 participants ( 5 males, 5 females).

A complete transcription of each focus group was performed, then content was analyzed to highlight the most relevant emerging topics.

\subsection{Results}

In the following, the main results are summarized for each UTAUT construct. The most significant quotations stated by the older adults in the study can be found in [26].

\subsubsection{Older Adults' Needs and TV Context of Use}

There is a feeling of melancholy, it is as if they were saying: "there is a gap that I have to fill". It is a sense of isolation, not so much real (presence of spouses, children, grandchildren...) as perceived, because they are now outside the work activity. This void is filled by the continuous presence of information (obtained mainly watching news TV programs) the link with the productive world, the "real" world.

The succession of the main news bulletins marks the day in its main moments, suggesting a regular routine. The information accompanies the day: in the morning people get up and turn on the TV to listen to the first news, they are updated at lunchtime, and then at dinner they watch the evening news.

If the need for information is the basis that unites participants, the choice of the preferred channel differentiates the cultural types present in the focus group, or the different channel demands. 
The most advanced range integrates the traditional/habitual reading of the daily newspaper on the Internet. At the opposite extreme are people who experience "nostalgia" when listening to the radio at any time or during any situation and who still live in a "classical" way (for these people, the modernization of the radio never happened). Then, there is the participant who "look on the world" and use satellite information to follow the news of other countries on CNN or BBC.

Television viewing takes place in different situations and within a specific social setting, which is most frequently the family.

Before the transition to DTT, television was watched in the family, within a circumscribed and known space, convenient, simple, and passive. When it was easier to get along, there was less choice and TV was watched together.

After the transition to DTT, television is watched alone, exploring new spaces, new channels in which one can also get lost. These are new viewing modalities separating the members of the family unit, bringing them to an enjoyment that is both individual and "scattered" among the various possible channels.

\subsubsection{Effort Expectancy}

Effort Expectancy in this case relates to the perceived ease of use of DTT and of the interaction through the remote controls.

The first answers to the questions of the interviewer on DTT can be described with the word "confusion." The main causes of this sense of confusion seem to be related to the interaction with remote controls. It is hard to find remote controls in the confusion of the different devices in the various rooms of the house.

There is even confusion about the existence of a "menu" that gives the possibility of receiving immediate information on programming. Participants are so confused such that someone thinks of the culinary sense of the word "menu."

As a result, the use of digital television requires commitment. This is not in line with the intrinsic nature of the television medium, which is mainly aimed at entertainment and relaxation.

Interaction with technology seems to generate a cognitive workload in older people, probably due to age-related visual, perceptive, psychomotor, and cognitive disabilities, where too many buttons, many channels, numbers to remember, etc. cause confusion. This often produces cognitive stress with fear, a sense of inadequacy, distance, and suspicion.

When asked what features of the new TV were a surprise, before mentioning the technical characteristics (increase in potential, higher number of channels, better reception), the participants answered: "How much it costs me!"

Clearly, participants are not talking about a simple financial cost, but rather a symbolic cost. This emotional cost is paid by those who have invested a curious interest in new technologies and by those who have tried to stay updated with modern times but have felt a sense of frustration and inadequacy from failing to do so. While all participants perceived the DTT offer to have great potential, they complained about the difficulty of exploiting it, leading them to give up and return to traditional TV with disappointment.

\subsubsection{Use Behavior and Attitude Towards DTT}

In this study, Use Behavior is related to the DTT usage by older adults. 
The change produced by DTT can generate two different types of reactions: acceptance or rejection.

Participants who accepted DTT and the new TV channels available in their daily choices. These participants tend to adopt a stimulating attitude towards technology and often take appropriate expedients to overcome problems. These people are the same using their exploratory skills on the Internet (reading online newspapers or looking for old friends on Facebook).

People who refuse DTT try to eliminate the changes produced by it, for instance, by selecting traditional channels and only viewing at those as if none others exist.

\subsubsection{Performance Expectancy}

Performance Expectancy relates in this case to perceived DTT usefulness.

Some immediate answers of participants during the focus group clearly show that nothing changed or improved after the introduction of DTT.

For the older adults, there is a lack of perceived advantages of DTT compared to traditional television. After some reflection later during the focus group, a few advantages were identified, such as a wider range of programs, available content, better resolution, and image quality.

More than half of those who were present (6 out of 10) claimed to have a PC at home.

Only some people stated to use the Internet routinely to read newspapers, keep in touch with friends on Facebook, watch a favorite TV broadcasts at the most convenient hours, write/receive emails, browse among the photos published by grandchildren on the network, or check their bank accounts.

In this segment, the PC was used mostly for emailing and Facebook as sources of communication and to attain human connection; it is no coincidence that this was especially prevalent among women participants.

The Internet is structural means for contact with the world that this group manifests. Wanting to be part of the modern day world is an indicator of openness towards what we call "new technologies," even in its new digital format, in terms of the Internet, social networks, TV, and Internet broadcasts.

\subsubsection{Facilitating Conditions and Social Support}

The switch-off representing the "point of no return" indicated the milestone where elders want to either establish themselves as a membership in the digitized society or not. The introduction of the DTT represents an event that involves the context of which one is a part of, first, the region then the nation. Therefore, the use of DTT builds an emotional feeling of belonging to society with respect to which, if you are not equipped, you can feel outside.

Participants in the focus group were critical towards the support provided to users during the transition process. Older adults were able to solve problems with DTT with the support provided by relatives and friends.

The implicit request that can be traced in the discursive flow of this group seems to support participants with further resources by simplifying modalities that can guarantee a better fruition.

The improvement proposals put forward by the group in the final phase of the focus group precisely lean towards improving the quality of the DTT offer. 


\section{Discussion}

From Field Study 1, it emerges that technology-resistant older adults are aware of the opportunities that such a new technological system can offer to improve their quality of life. The EasyReach system was overall perceived as useful for social interaction (Performance Expectancy). The pleasantness (Hedonic Motivation) and ease of use (Effort Expectancy) were also carefully examined as potential factors that may affect acceptance.

In accordance with other works [17], the results of this investigation suggest that the use of traditional devices to interact with digital environments can lower the risk of rejection by technology-resistant participants and, especially for aged people, could provide a solution to mitigate social isolation. The use of familiar devices (such as TV sets) in the interaction should improve the acceptance of ICT services by older adults.

By engaging older adults in real-life scenarios that did not require direct interaction, this work shows a large collection of opinions and sentiments about a realistically perceived opportunity. Further investigations are needed to assess direct interactions in and evaluate user acceptance in a real-life environment.

The quantitative exploratory Field Study 2 allowed the authors to investigate factors facilitating or hampering the acceptance of an Electronic Health Record by senior citizens and also to compare the results obtained for the overall sample of citizens and for the group of adults over 55 .

Performance Expectancy and Facilitating Conditions appear to be the most relevant determinants of Behavioral Intention to use the TreC system. Performance Expectancy is connected to the perceived usefulness of the TreC system by senior citizens in daily life, improvements in the health management, and opportunities provided by the system to save time. Facilitating Conditions include the availability of technological resources necessary to use TreC, knowledge (skills) necessary to use TreC, and necessary help/support available when difficulties arise during usage. Minor importance appears to be devoted to Effort Expectancy, reflecting both the ease of learning how to use the system at the beginning and ease of use after the first period. In other studies, an important role was devoted to Effort Expectancy in promoting acceptance, especially in the early stages of introducing the service. The less important role of Effort Expectancy in this study, in comparison with other studies [5], can be ascribed to the characteristics of the sample, which was composed by people skilled in technology use and that utilize Internet services in their daily lives.

Overall, the investigation on acceptance of older adults over 55 years of age showed no big differences between general model variables, including all participants. Performance Expectancy scores and Facilitating Conditions seem to be the most relevant determinants of Behavioral Intention to use the TreC system.

This field study was realized by involving older adults who have a high education (degree or post degree), are skilled in technology use, and employed. Further research should focus on the acceptance of TreC services by older adults who have different characteristics, such as less technological skill and lower education.

The results of the TreC field study suggest that public policies to promote acceptance of innovative e-health solutions by older adults should be mainly focused on:

- Increasing the perceived usefulness of the new solutions (for instance, setting up adequate communication campaigns to promote awareness about services made available by EHR); 
- Providing adequate support for facilitating the utilization of health services (such as with online help, a website, or contact center).

The results of Field Study 3 on the acceptance of digital television by older adults suggest that the behavior of the elderly group is not homogeneous. The transition to DTT induced many different emotional reactions. In one way, the innovation was viewed as a menace or too much of a change in how television is traditionally watched on a daily basis, while another reaction was that the innovation was a desirable change and presented a development opportunity.

Each event, concerning the presentation of the technology, received different emotional constructions by each person who is in the same environment and acts in it. From the introduction of DTT, different groups projected their own desires and fears based on learning and acceptance the new technology. Acceptance attitudes (from participants who decided to explore new channels) or rejection attitudes (from those who tried to reset the change by reprogramming the old channels, acting ignorant towards new channels) were defined by the different and specific emotional representations, or cultures, of the analyzed event. In the moments of intense change, the cultural models were revealed with defined clarity. Reactions to the change towards DTT and the relationship with the new telecommunication technologies depend on, and simultaneously reveal, the different cultures of participants.

It was also found that the fear of learning DTT and new ICT technologies prevails against curiosity, or vice versa, to the extent that these two sub-groups are identified based on the following cultural characteristics:

- Fear: Participants fearful of DTT and new technologies changes exhibit a sense of inadequacy, incompetence, and clumsiness.

- Curiosity: Personal interests and passions of older adults in this subclass, who are unskilled in new technologies, allow them to overcome the psychological and physical barriers due to age. This group can be a powerful basis for word-of-mouth, horizontal peer-to-peer promotion and personalized help to complement the support provided by customized help groups and call centers.

\section{Limitations of the Study}

In the field studies, the cognitive impairment level of the recruited older people was not considered. Therefore, the influence of declining cognition on ICT acceptance by older people should be investigated in future studies.

Another limitation is that the factors affecting older adults to accept various ICT services (such as TV-based technology for social interaction, an Electronic Health Record, and Digital Television) were all analyzed within the same reference framework (Unified Theory of Acceptance of Use of Technology). Also, the users involved in the three studies had different socio-demographic characteristics, while different methods were adopted for data collection (i.e., quantitative or qualitative). Consequently, it is not possible to compare the results obtained in the three field studies; thus, only general considerations can be suggested about ICT acceptance by older adults.

Geographical localization of the three field studies, which were performed in Italy, is another limitation of this study. While the analyzed methods could have potential in many different populations and countries, the obtained the results can only be specified to senior citizens in Italy. 


\section{Conclusions}

In order to identify the main predictors of acceptance of ICT services by older adults, the UTAUT and UTAUT2 models were quantitatively and qualitatively employed in three field studies. Overall, results confirm that the reference model variables represent the key factors for older adults in predicting their viewpoint toward ICT.

Performance Expectancy and Facilitating Conditions seem to be the most relevant factors in all field studies. Also, the relevance of Hedonic Motivation emerged clearly. The role of ease of use seems to be affected by the user profiles, which were based on education level, experience with technology, and employment. For instance, in Field Study 2, Effort Expectancy was found to have less importance, most likely due to the characteristics of participants who were skilled in technology use and utilize daily Internet services.

Another important factor overcoming barriers preventing the acceptance of ICTs by older adults is the use of familiar devices (as TV sets) in the interaction, as suggested by Field Study 1.

From Field Study 3, it was apparent that older adults' acceptance/rejection of ICT services depends on classical socio-demographic variables (age, gender, level of education, number of family members, composition of the household, and geographical area) and on specific "cultures," including attitudes towards technology, lifestyle, and beliefs that individuals share with members of their social context.

\section{Author Contributions}

The authors contributed equally to this work.

\section{Competing Interests}

The authors have declared that no competing interests exist.

\section{References}

1. Loos E, Haddon L, Mante-Meijer E, editors. Generational Use of New Media. Ashgate; 2012

2 ISTAT, Cittadini, imprese e ICT, Report; 2017. Available from: https://www.istat.it/it/files//2017/12/ICT_Anno2017.pdf

3 Gomes G, Duarte C, Coelho J, Matos E. Designing a facebook interface for senior users. The Scientific World J. 2014; 2014: 741567. doi: 10.1155/2014/741567.

4. European Commission. Digital agenda for Europe. Luxembourg: Publications Office of the European Union; 2014.

5. Venkatesh V, Morris MG, Davis GB, Davis FD. User acceptance of information technology: Toward a unified view. MIS Quart. 2003; 27: 425-478.

6. Loos E, Haddon L, Mante-Meijer E, editors. The social dynamics of information and communication technology. Ashgate; 2008.

7. Silverstone R, Haddon L. Design and the domestication of information and communication technologies: Technical change and everyday life. Oxford University Press; 1996.

8. Haddon L. Roger silverstone's legacies: domestication. New Media and Society. 2006; 9: 25-32. 
9. Norman DA. Design of everyday things: revised and expanded. London: MIT Press (UK edition); 2013.

10. Hassenzahl M, Diefenbach S, Goritz, A. Needs, affect, and interactive products - Facets of user experience. Interact Comput. 2010; 22: 353-362.

11. Sapio B, Turk T, Cornacchia M, Papa F, Nicolò E, Livi S. Building scenarios of digital television adoption: a pilot study. Technol Anal Strat Manage. 2010; 22: 43-63.

12. Venkatesh $\mathrm{V}$, Thong J, Xu X. Consumer acceptance and use of information technology: Extending the unified theory of acceptance and use of technology. MIS Quart, 2012; 36: 157178.

13. Papa F, Nicolò E, Livi S, Sapio B, Cornacchia M. Factors affecting the usage of payment services through digital television in Italy. Proceedings of European Interactive TV and Video (EurolTV 2010) Conference; 2010 June 9-11; Tampere Finland.

14. Newell AF, Carmichael A, Gregor P, Alm N. Information technology for cognitive support. In: Jacko JA, editor, The human-computer interaction handbook: fundamentals, evolving technologies and emerging applications. Lawrence Erlbaum Associates, Inc.; 2003. 464-481.

15. Papa F, Cornacchia M, Sapio B, Nicolò E. Engaging technology-resistant older adults: Empirical evidence from an ICT-enabled social environment. Inform Health Soc Care. 2017; 42: 18. doi: 10.3109/17538157.2016.1153477.

16. Kamel Boulos MN, Lou RC, Anastasiou A, Nugent CD, Alexandersson J, Zimmermann G, et al. Connectivity for healthcare and well-being management: examples from six European projects. Int J Env Res Pub Heal. 2009; 6: 1947-1971.

17. Alaoui M, Lewkowicz M. Struggling against social isolation of the elderly - The Design of SmartTV Applications. Proceedings of the 10th International Conference on the Design of Cooperative systems; 2012 May 30-June 1; London: Springer.

18. Correia L, Costa N, Pereira A. Fighting elders' social and technological exclusion: the TV based approach. 5th International Symposium on Ambient Intelligence; 2014 04-06 June; Salamanca. London: Springer.

19. Bisiani R, Merico D, Pinardi S, Dominoni M, Cesta A, Orlandini A, et al. Fostering Social Interaction of Home-Bound Older adults: The EasyReach System. In: Ali M, Bosse K, Hindriks V, Hoogendoorn M, Jonker CM, Treur J, editors. Recent Trends in Applied Artificial Intelligence. Springer; 2013. (33-42).

20. Nielsen J. Seniors as Web Users. Nielsen Norman Group; 2013 May 28. Available from: http://www.nngroup.com/articles/usability-for-senior-citizens/.

21. Nielsen J. Authentic Behavior in User Testing. Nielsen Norman Group; 2005 February 14. Available from: http://www.nngroup.com/articles/authenticbehavior-in-user-testing/.

22. Papa F et al. Detailed Plan for the Pilots. Technical Report. EasyReach Project; 2012; Deliverable D6.1

23. Tang PC, Ash JS, Bates DW, Overhage JM, Sands DZ. Personal health records: Definitions, benefits, and strategies for overcoming barriers to adoption. J Am Med Inform Assoc. 2006; 13: 121-127.

24. Zanutto A. "Two Clicks and I'm in!" Patients as co-actors in managing health data through a personal health record infrastructure. Health Inform J. 2017; doi 10.1177/1460458217712056.

25. Papa F, Zanutto A, Nicolò E, Sapio B. Measuring citizens' acceptance of an electronic health record in Italy. Int J Healthc Technol Manage. 2019 
26. Papa F, Sapio B, Pelagalli M F.User experience of older adults with digital television. Proceedings of the 9th international conference on Interactive television (EurolTV '11); 2011 June 29 -July 1; Lisbon Portugal

27. Papa F. Analisi delle esperienze d'uso (user experience) con la televisione digitale terrestre nei principali Paesi Europei. Technical report. Fondazione Ugo Bordoni; 2010

28. Madden M. Older adults and social media: Social networking use among those ages 50 and older nearly doubled over the past year. Technical report. Pew Research Center; 2010

29. Verdegem P, Hauttekeete L, De Marez L. The analogue switch-off in a cable dominated television landscape. Implications for the transition to digital television in Flanders. Communications, the European Journal of Communication Research. 2009; 34: 87-101.

30. Carli R, Paniccia RM, Lancia F, Pelagalli MF, Nuovi modelli di comunicazione e sviluppo territoriale. Psicologia Clinica. 1997; 2: 41-64.



Enjoy OBM Geriatrics by:

1. Submitting a manuscript

2. Joining in volunteer reviewer bank

3. Joining Editorial Board

4. Guest editing a special issue

For more details, please visit: http://www.lidsen.com/journals/geriatrics 